\title{
Impact of fast vertical and radial plasma movement on type-I ELMs in ASDEX Upgrade
}

\author{
P.T. Lang, O. Gruber, S.H. Kim*, J.B. Lister*, Y.R. Martin*, S.Yu. Medvedev ${ }^{\dagger}$, V. Mertens, \\ R. Neu, F. Ryter, W. Schneider, A.C.C. Sips, W. Suttrop, ASDEX Upgrade Team \\ Max-Planck-Institut für Plasmaphysik, EURATOM Association, \\ Boltzmannstr. 2, 85748 Garching, Germany \\ * CRPP-EPFL, Association EURATOM-Confédération Suisse, 1015 Lausanne, Switzerland \\ $\dagger$ Keldysh Institute for Applied Mathematics, Russian Academy of Sciences, Moscow, Russia
}

\section{Introduction}

The choice of the type-I ELMy H-mode as the standard operational scenario for ITER creates a dilemma. On the one hand, high confinement requires rather low ELM frequencies $f_{E L M}$. On the other hand, maximum acceptable heat loads onto in-vessel components during ELMs can be only achieved by operating at sufficiently high $f_{E L M}$ when the energy expelled from the plasma per ELM, $\Delta W_{E L M}$, which satisfies $\Delta W_{E L M} \propto 1 / f_{E L M}$ [1], drops to sustainable levels [2]. In case this scenario is operated hence a technique for ELM control is required. A possible approach, currently under investigation is ELM suppression by edge resonant magnetic field perturbation [3] or ELM mitigation by pellet pacing [4]. To widen the latter approach, also alternative pacing methods are under investigation. For their aptitude it is of interest which operational range can be covered but also which unwanted inherent side effects are involved. From the validated methods the best one(s) can be selected to serve in ITER.

We investigate the approach of the "magnetic triggering", a technique first suggested and demonstrated at TCV [5]. TCV attributed the observed effect of locking the ELM time sequence with a fast plasma oscillation to an edge current induction during the motion as the vertical movement takes place in a spatially inhomogeneous singlenull vacuum field configuration. The suitability of a fast vertical plasma motion for ELM pacing was confirmed in ASDEX Upgrade and demonstrated for a clear type-I ELMy H-mode discharge as well [6]. However, it was also understood that the interpretation of the observed behaviour is not simple and straight forward but requires detailed modelling taking into account essential features of the magnetic configuration and the vessel structure. As a consequence, analysis has been performed proposing several different physics approaches. The quasi-equilibrium plasma evolution modelling using the DINA-CH code demonstrated that the massive copper passive stabilization loop (PSL) inside the vacuum vessel of ASDEX Upgrade can produce an external flux linking change similar to those created in TCV by the vertical movement [7]. A further explanation is the flux surface deformation pattern with increasing local plasma boundary squareness during the plasma downward motion due to the presence of PSL. The calculations with the finite element stability code KINX taking into account the separatrix suggested that destabilization of medium-n edge kink-ballooning modes due to the plasma shape deformation were expected to trigger ELMs [8]. For validation of the given explanation experiments with a radial plasma movement have been proposed for ASDEX Upgrade predicting a similar influence on ELMs like the vertical motion [7]. 


\section{Set up}

The task of this approach was the experimental test of this prediction and compare fast vertical and radial plasma motion with respect to their ability to control the frequency of ELMs. Again, as for our previous study on the vertical motion [6] we relied on the feedback controlled plasma position and shape control system [9]. Actuators are 10 poloidal magnetic field (PF) coils and eight vertical field coils (20 ms response time) located outside the toroidal magnetic field (TF) coil system and distant from the plasma. As control parameters we have chosen (again) the centre of current (2nd order regression analysis) indicated as "z" and the outermost radial plasma position "R". For the driving frequency we selected $f_{D}=50 \mathrm{~Hz}$ ( $40 \mathrm{~Hz}$ in a few cases as well) since resonant excitation of the whole vessel or partial structures becomes dangerous for lower frequencies while higher frequencies result in a reduction of the amplitude obtained with respect to the requested one.

Since ASDEX Upgrade en route to an all Tungsten (W) device has now most of the divertor and main chamber plasma facing components covered by $\mathrm{W}$ [10], the operational behaviour has altered since our previous "magnetic triggering" experiments. In particular, it turned out more troublesome to achieve stable operation in the type-I ELM regime with a low ELM frequency. Therefore, we applied a different configuration in this study, a deuterium plasma discharge in lower single null (LSN) configuration with $I_{P}=800 \mathrm{kA}, B_{t}=-2.4 T$ (counter clockwise direction from above defining positive values, $I_{P}$ "technical" or ion direction), $q_{95}=5.5$, elongation $\kappa=1.77$, upper and lower triangularity of $\delta^{u}=0.11$ and $\delta^{l}=0.38$, respectively. Auxiliary heating by $D^{0}$ neutral beam injection (NI) with a power of $P_{N I}=5.0 \mathrm{MW}$ transferred the discharge into the H-mode with a reasonable intrinsic ELM frequency $f_{E L M}^{0}$, central electron cyclotron resonance heating (EHRH, $400 \mathrm{~kW}$ at $140 \mathrm{GHz}$ ) to counteract core impurity accumulation. Unfavourably the vacuum vessel conditions changed rather fast after each boronisation preventing momentarily strong impurity influx and as a consequence evolution of discharges run with identical settings changes gradually but also individual discharges unveiled a transient behaviour like a gradual increase of $f_{E L M}^{0}$.

A poloidal cross section of ASDEX Upgrade hosting the applied configuration is shown in figure 1. In the C2006 experimental campaign an almost full $\mathrm{W}$ coverage of the plasma facing components was reached, with the exception of only a few carbon (C) tiles in the divertor (indicated by grey filling) and at the ion cyclotron (IC) antennae guard limiters (dash black). The upper and lower PSL contour is shown in black, the major effect is attributed to the upper one due to its closeness to the plasma surface [7, 8]. Furthermore, the $\mathrm{R}$ and $\mathrm{z}$ locations are shown as well as the quantity "Gap", the radial distance of the separatrix at the top of the IC antennae. As well, some lines of sight are displayed for the line averaged density $\bar{n}_{e}$ as measured by the DCN laser interferometer and the soft x-ray (SXR) cameras. Finally, the observation region of the $D_{\alpha}$ radiation ELM monitor (displaying the impact of the ELM induced pressure pulse in front of the outer divertor strike point) and two channels of the electron cyclotron emission (ECE) radiometer measuring the electron temperature $T_{e}$ (distance $20 \mathrm{~mm}$ ) are given.

Based on a database of pre-calculated equilibria, the magnetic measurements are used to calculate the magnetic configuration and several plasma parameters such as geometrical elements (x-point positions, uppermost and outermost point of separatrix, current centre, "Gap" etc.) and the MHD plasma energy $W$ by statistical regression (FP:= Function Parameterization) [11]. These data are normally acquired at a rate of $1 \mathrm{kHz}$ and made available routinely 


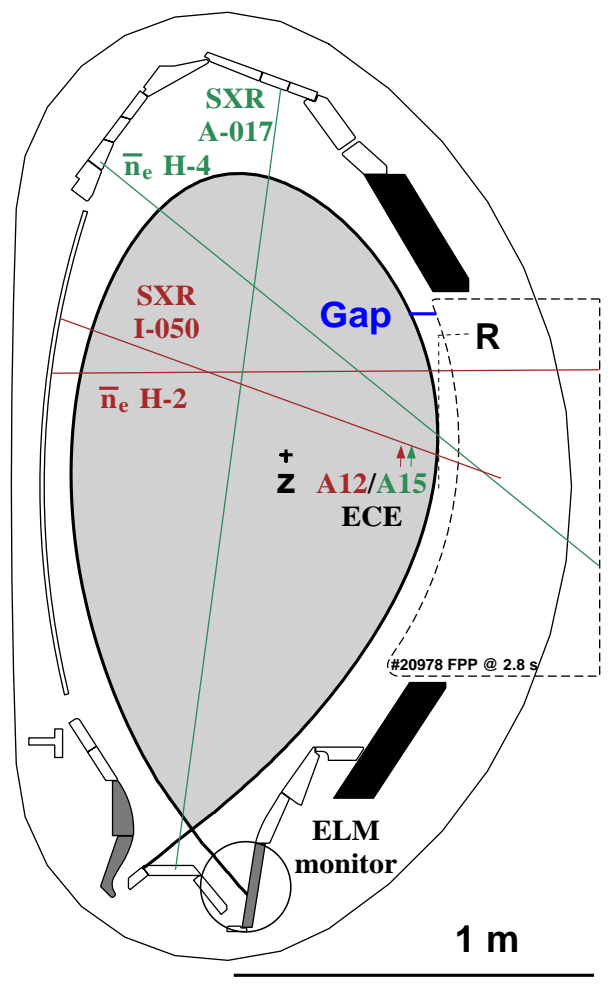

Figure 1: Poloidal cross-section of the tokamak ASDEX Upgrade, typical plasma equilibrium applied, location of relevant diagnostics and geometric quantities.

for the entire discharges. For dedicated phases equilibria (and some geometrical elements) were reconstructed with CLISTE [12], an interpretive equilibrium code.

\section{Results}

For the comparison of the impact of an imposed vertical and radial motion on the ELMing behaviour, we first checked the realised plasma motion. The discharge shown in figure 2 had two motional phases, one with a pre programmed vertical (left section) and one with a radial (right section) movement. In between, a quiescent intrinsic reference phase (middle section) was placed. For each phase a $100 \mathrm{~ms}$ long period was selected for CLISTE analysis, $80 \mathrm{~ms}$ sequences thereof displayed.

The lowermost trace shows the ELM monitor signal, above the "z" position of current centre and the outermost separatrix position "R" are plotted. In red, requested values are displayed, blue curves refer to reconstructed PF values while black lines are obtained by CLISTE. The "Gap" position (FP only) is the closest indicator for the plasma separatrix motion with respect to the relevant upper PSL. Further upwards in each case two signals from different SXR, ECE and DCN interferometer channels are plotted, the corresponding positions can be seen in figure 1. Obviously, for both motional phases the requested motion is achieved, however with some phase shift and amplitude reduction. Phase shift and amplitude reduction are more pronounced for the vertical case, hence the control system response time is less for the radial motion. Although CLISTE resolves the ELM induced plasma motion more clearly and seems to have better temporal resolution (less phase shifted achieved motion) also the 


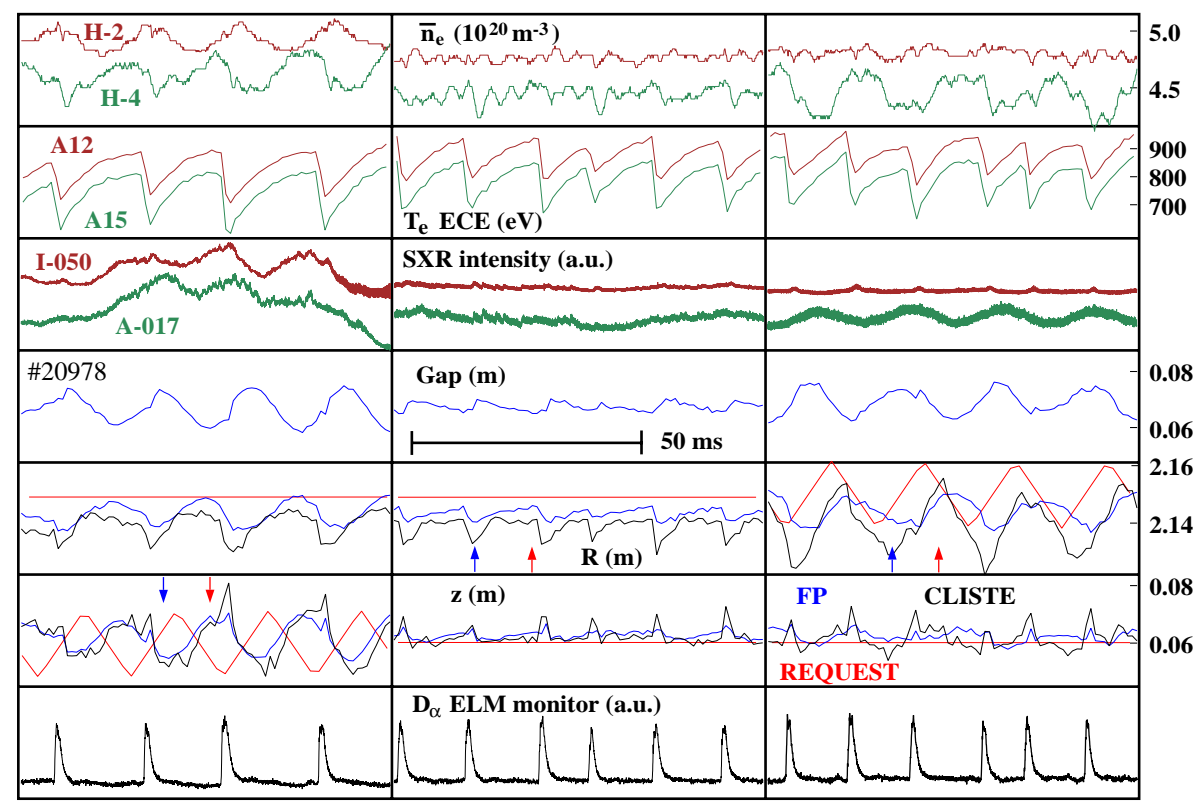

Figure 2: Sequences with vertical (left) and radial motion applied (right), reference phase (middle). From top: density, temperature, SXR signal evolution at positions or along lines-of-sight as indicated in figure 1. Derived values for "Gap", outermost plasma position and current centre (requested values in red, FP data in blue and CLISTE in black). ELM monitor signal (observation area indicated in figure 1).

FP reconstruction reproduces the plasmas motional behaviour quite well. Available for any time and reasonably reflecting the plasma motion, the FP data thus were applied for further analysis of the ELM frequency locking behaviour. The demanded motion is confirmed as well by the diagnostics, most obviously by the $\bar{n}_{e}$ evolution observed with the DCN laser interferometer. The channel H-2 essentially visualises the vertical, H-4 the radial motion. The upward, respectively outward motion shift regions of higher density into the line of sight raising the signal there and vice versa. Some distortion by the ELM is visible as well since each event causes a down/in kick of the plasma due to the induced pressure loss and hence reduced Shafranov shift. As well, this pressure loss is created by a drop of electron density and temperature particularly in the edge region. This $T_{e}$ drop dominates the evolution of the temperature signal recorded by ECE. The selected distance between the two channels used was about the same as the amplitude of the full R motion. Thus it becomes obvious the effect of the local ELM induced temperature drop is about 4 times stronger than the impact imposed by the motion. Regardless the influence of moving R remains visible by a steepened/flattened post ELM increase of $T_{e}$ during phase with outward/inward motion. Finally, the plasma motion is visible from the SXR data as well, evidently in the radial case, somewhat superposed by transient plasma evolution in the vertical case.

For each of the three phases, two time points were selected referring to extreme positions of the motion. These times are marked by the arrows in figure 2 , and the corresponding equilibria are plotted in figure 3 . It displays separatrix contours calculated by CLISTE and as reference a common one from FP. CLISTE equilibria show the upper and lowermost plasma position in the vertical phase (left), the outer and innermost in the radial phase (right) and the situation just after and just before an ELM (middle). The vertical motion essentially creates a 


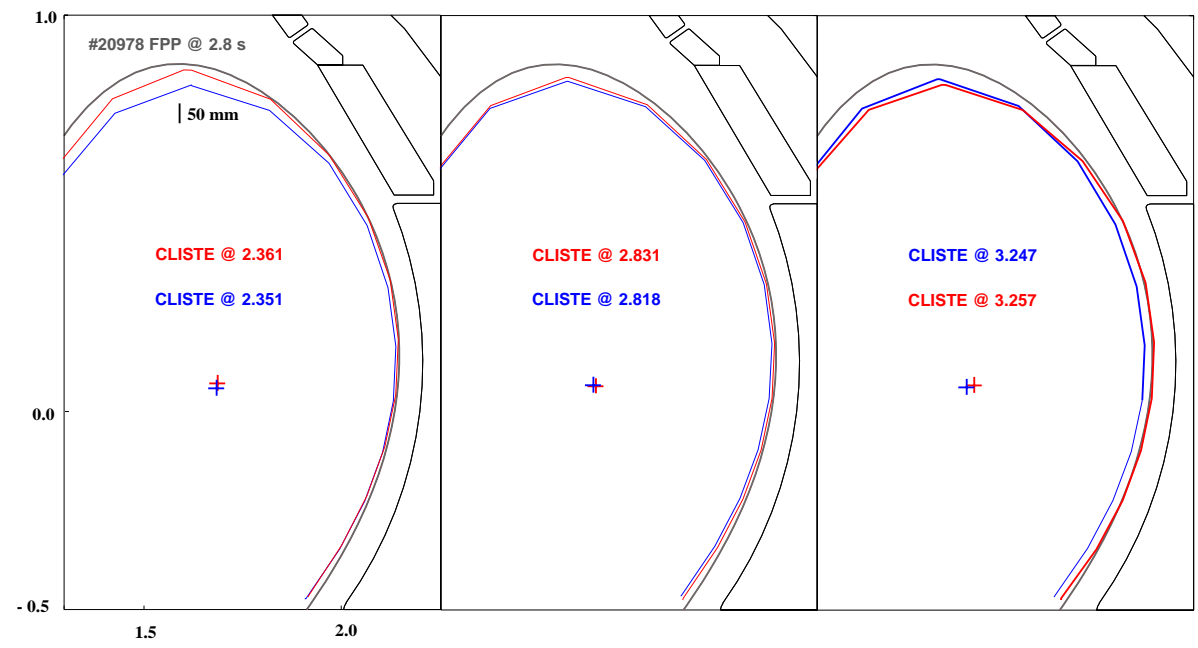

Figure 3: Extreme positions for vertical (left) and radial (right) motion and for an intrinsic ELM (middle). Plasma contours for times indicated by arrow in figure 2 as obtained by CLISTE, a reference FP equilibrium is indicated as well.

more elongated plasma since the separatrix sweeps mostly at the upper boundary. There is almost no change in the vicinity of the X-point. Correspondingly, the radial motion essentially changes the outermost separatrix position by creating a somewhat fatter plasma. Here, the plasma column is tilted slightly clockwise during the motional cycle. Separatrix motions with respect to the upper PSL predicted essential for the ELM triggering due to the corresponding stability boundary change $[7,8]$ are of the same amplitude during vertical and radial phases. Anyway, for both cases of imposed plasma motion the created change of the plasma edge region contour is more pronounced than the effect stemming from the ELM itself. This can be seen clearly when comparing to the slight shrinking of the confined plasma cross section caused by the ELM. Sweeping amplitudes in the range of the ELM imposed ones were found critical in order to achieve locking effects in our previous study. Hence, with respect to this criterion the realized plasma motion can be regarded of sufficient amplitude to probe its appropriateness for ELM pacing.

Having assert a sufficient plasma sweep amplitude is created, we investigated now the influence of a radial motion on the ELM cycle in comparison to the vertical "magnetic triggering" approach. Therefore, we compared phases in two otherwise identical set up discharges, one with a $700 \mathrm{~ms}$ long vertical, the other one with an according radial motion. The result is shown in figure 4. Again, like in the examples shown in figure 2, the peak-to-peak amplitude of the requested motion was chosen $24 \mathrm{~mm}$. In the early phases where the motion was imposed these discharges developed reasonable low intrinsic ELM frequencies close to $50 \mathrm{~Hz}$ however gradually increasing. Both discharges seems to develop a slightly different background behaviour, the first one (vertical motion) showing tendency to ramp $f_{E L M}^{0}$ from about $40 \mathrm{~Hz}$ at $1.8 \mathrm{~s}$ to about $70 \mathrm{~Hz}$ at $2.8 \mathrm{~s}$. The second one (vertical motion) showed a more stable $f_{E L M}^{0}$ value slightly above $50 \mathrm{~Hz}$. Obviously, the second one yields the better target for frequency locking at $f_{D}=50 \mathrm{~Hz}$ anyway.

The vertical motion (left part of figure 4) initiates ELM frequency locking and hence successful "magnetic trig- 


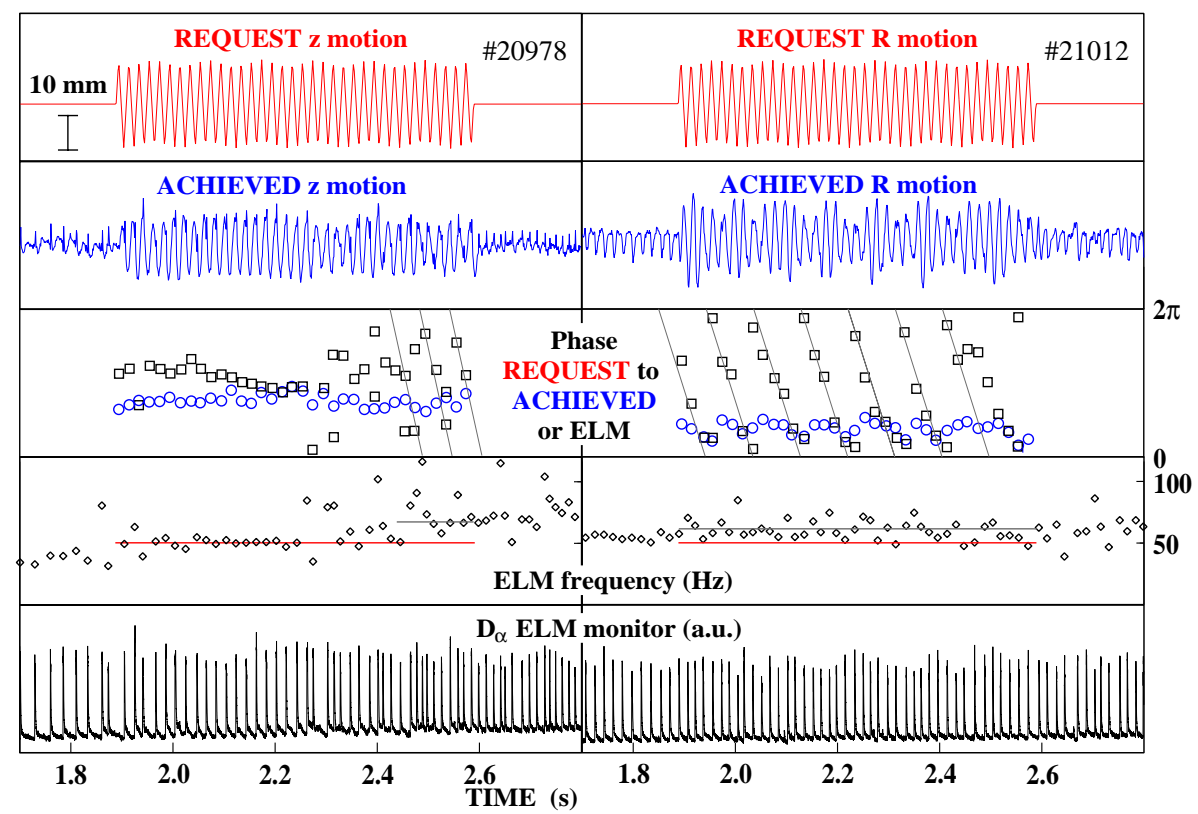

Figure 4: Strong locking attempts with vertical (left) and radial (right) motion. From top: requested motion; achieved motion (FP); phase of achieved motion (circles) and ELM onset (squares) with respect to requested motion (zero defined by uppermost/outermost requested position), ELM frequency and monitor. Frequency locking is achieved in the first part of the vertical motion sequence but lost later with the intrinsic ELM frequency too different to the driving frequency (red solid line). No locking is observed for the radial motion, ELM and motion frequency do not affect each other than creating a beat wave.

gering" right at the beginning of the sweeping phase shifting $f_{E L M}^{0} \approx 40 \mathrm{~Hz}$ to $f_{E L M}=f_{D}=50 \mathrm{~Hz}$. Here, the same behaviour as that reported in [4] is observed namely the ELMs are triggered by the downward motion of the plasma. This can be seen from the phase relation shown in figure 4. Relevant parameters are displayed (top to bottom) the requested and achieved (vertical or radial) motion, the phase delay between achieved and requested motion (circles) and between ELM onset to requested motion (squares, zero phase defined by requested position most upper/outwards), ELM frequency (red: $f_{D}=50 \mathrm{~Hz}$, grey: intrinsic averaged ELM frequency, the according ELM delay phase evolution is indicated with the phase data as well) and ELM monitor. (It should be noted taking the turning points of the pre-programmed requested motion were chosen as reference point since these times are stored and this evolution is of course unaffected by the ELM itself. This way, phasing analysis showed best performance.) Thus, the ELM onset delayed by about $\frac{\pi}{2}$ with respect to the achieved uppermost position (which is in turn delayed by about $\frac{\pi}{2}$ with respect to the requested uppermost position) means that the magnetic ELM triggering takes place halfway between upper and lowermost position at the time with the fastest downward motion. Gradually along the $\mathrm{z}$ motion phase, this delay shrinks until at about TIME $=2.2 \mathrm{~s}$ the ELM occurs already shortly after the plasma passed through its uppermost $\mathrm{z}$ position (the pulling of the achieved phase towards the ELM phase is induced by the up kick of the plasma by the ELM now taking place very close to the uppermost position and hence the ELM maximum now exceeding and masking the imposed motional maximum). At the time the ELM occurrence starts to coincide with the uppermost plasma position, the ELM frequency locking gets lost and after 
about TIME $=2.3 \mathrm{~s}$ an intrinsic ELM frequency of about $68 \mathrm{~Hz}$ is established. This can be concluded from the regular phase shift of the ELM after TIME $=2.4 \mathrm{~s}$ until the end of the imposed motion phase, the grey line plotted in the phase signal box corresponds to an average $f_{E L M}^{0}$ value as represented by the grey line in the ELM frequency signal box. Obviously, for the $\mathrm{z}$ amplitude applied here ELM frequency locking can be achieved only in a narrow frequency band around $f_{D}$, here ranging from about 40 to $65 \mathrm{~Hz}$.

No indication for a frequency locking of the ELMs to the imposed motion was found for the radial movement. The intrinsic frequency evolves with about $60 \mathrm{~Hz}$ all along the motional phase. This is clearly displayed by the regular phase shift of the ELMs with respect to imposed and realised motion. Again, the grey line shown in the

ELM frequency box $\left(f_{E L M}^{0} \approx 60 \mathrm{~Hz}\right)$ corresponds to the evolution of the phase shift indicated by the grey line in the phase signal box. Achieved motion and ELM activity evolve independent (except the phase pulling artefact in the analysis) from each other but as both impose some $\mathrm{R}$ motion there is some interference taking place. With a beat wave frequency of about $14 \mathrm{~Hz}$ alternating constructive and destructive interference between both motional components can be observed, yielding phases with enhanced and reduced overall motion.

\section{Conclusions}

Experiments have been performed imposing a fast cyclic plasma motion in vertical or radial direction during phases with reasonably low and stable type-I ELMs. For the verified amplitudes of the motion initial theoretical investigations predicted magnetic triggering as well for a radial excitation. Frequency locking could be confirmed for the vertical case despite the fact that target discharges were less suitable due to the larger area inside the vessel covered by W. At an amplitude of $24 \mathrm{~mm}$ and a driving frequency of $50 \mathrm{~Hz}$, frequency locking could be established from intrinsic ELM rates in the range 40-65 Hz, larger frequency deviations results in a loss of the locking. Delocking in the case of a gradual slip of $f_{E L M}^{0}$ out of the capture range is observed to correlate with a phase shift from the fastest plasma downward motion back towards the plasma uppermost position. For radial motion, in the applied parameter range ( $f_{D}=4050 \mathrm{~Hz}$, up to $24 \mathrm{~mm}$ amplitude) no frequency locking of ELMs could be observed. More refined additional stability analysis of the ELM triggering sequences with radial plasma movement in ASDEX Upgrade suggests the following explanation of the achieved experimental results: the plasma boundary deformation pattern during the radial movement does not provide the sufficient change in the high- and medium-n mode stability limits despite the amplitude of the deformation comparable to the vertical movement case.

\section{References}

[1] HERRMANN, A., Plasma Phys. Controlled Fusion 44 (2002) 883.

[2] FEDERICI, F. et al., Plasma Phys. Controlled Fusion 45 (2003) 1523.

[3] MOYER, R. A. et al., Phys. Plasmas 12 (2005) 056119.

[4] LANG, P. T. et al., Nucl. Fusion 43 (2003) 1110.

[5] DEGELING, A. et al., Plasma Phys. Controlled Fusion 45 (2003) 1637.

[6] LANG, P. T. et al., Plasma Phys. Controlled Fusion 46 (2004) L31.

[7] KIM, S. H. et al., 32 ${ }^{\text {nd }}$ EPS Conf., Tarragona 2005, P5.014 . 
[8] MEDVEDEV, S. Y. et al., 32 ${ }^{\text {nd }}$ EPS Conf., Tarragona 2005, P5.064 .

[9] MERTENS, V. et al., Fusion Science and Technology 44 (2003) 593.

[10] NEU, R. et al., Nucl. Fusion 45 (2005) 209.

[11] SCHNEIDER, W. et al., Fusion Eng. Design 48 (2000) 127.

[12] MCCARTHY, P. J. et al., ELM-resolved interpretive MHD equilibria on ASDEX Upgrade using SOL tile currents and kinetic data, in Europhysics Conference Abstracts (CD-ROM, Proc. of the 30th EPS Conference on Controlled Fusion and Plasma Physics, St. Petersburg, 2003), edited by KOCH, R. and LEBEDEV, S., volume 27A, pages P-1.64, Geneva, 2003, EPS. 